\title{
Bioinformatics and functional magnetic resonance imaging in clinical populations: practical aspects of data collection, analysis, interpretation, and management
}

\author{
Diana J. Vincent, Ph.D., And Mark W. Hurd, Ph.D. \\ Departments of Radiology and Neurosciences, Medical University of South Carolina, Charleston, \\ South Carolina; and Department of Psychology, College of Charleston, South Carolina
}

\begin{abstract}
In this paper the authors review the issues associated with bioinformatics and functional magnetic resonance (fMR) imaging in the context of neurosurgery. They discuss the practical aspects of data collection, analysis, interpretation, and the management of large data sets, and they consider the challenges involved in the adoption of fMR imaging into clinical neurosurgical practice. Their goal is to provide neurosurgeons and other clinicians with a better understanding of some of the current issues associated with bioinformatics or neuroinformatics and fMR imaging. Thousands to tens of thousands of images are typically acquired during an fMR imaging session. It is essential to follow an activation task paradigm exactly to obtain an accurate representation of cortical activation. These images are then interactively postprocessed offline to produce an activation map, or in some cases a series of maps. The maps may then be viewed and interpreted in consultation with a neurosurgeon and/or other clinicians. After this consultation, long-term archiving of the processed fMR activation maps along with the standard structural MR images is a complex but necessary final step in this process. The fMR modality represents a valuable tool in the neurosurgical planning process that is still in the developmental stages for routine clinical use, but holds exceptional promise for patient care.
\end{abstract}

KEY WORDS • bioinformatics • functional magnetic resonance imaging • neurosurgery

The introduction of fMR imaging more than a decade ago has served as an important tool for understanding how the brain functions. Although fMR imaging is still a relatively young field, in several good general review articles the theoretical, methodological, and interpretive challenges in this multidisciplinary field have been discussed., ${ }^{1,2,7,8}$ Additionally, despite its reputation as primarily an experimental tool, in several papers it has been suggested that fMR imaging may be a valuable adjunct tool for neurosurgery if used in concert with other mapping techniques. ${ }^{4,10,12,17,18,23}$ As the use of fMR imaging continues to grow beyond experimental populations to clinical applications in patient populations, there are many practical challenges associated with data collection, analysis, interpretation, and management in this context.

\section{BIOINFORMATICS AND fMR IMAGING}

In this paper, we present a brief overview of informaticsrelated issues within the context of fMR imaging, with a specific focus on neurosurgery. We reference both the current literature and our experience of more than a decade in developing various aspects of this modality in a clinical set-

Abbreviations used in this paper: $\mathrm{fMR}=$ functional magnetic resonance; MAIAL = Multidisciplinary Advanced Image Analysis Laboratory; RAID = Redundant Array of Inexpensive Discs. ting. During these years we have worked closely with neurosurgeons on a variety of cases from tumor resection, epilepsy surgery, electrode grid implantation for chronic pain, and radiation therapy, to the importation of activation maps into surgical navigational systems. Additionally, we have built and maintain a university-wide network of servers and workstations to process and archive fMR imaging data for both experimental and clinical purposes.

In the context of this modality and neuroinformatics, there are a number of considerations that should be made before requesting an fMR imaging study as part of the planning process for a surgical intervention..$^{18}$ An fMR imaging study essentially involves a data collection phase in the magnet, an offline postprocessing phase for data analysis and interpretation, and a data management phase $;{ }^{14}$ each of these phases will be discussed in turn. It is important to note, however, that it is generally difficult to request an fMR imaging study and immediately obtain images with areas of activation mapped to specific regions of the brain. Furthermore, accessing fMR images afterward can often be difficult because of current incompatibilities between software packages. ${ }^{18}$ Unlike standard clinical neuroimaging modalities (computerized tomography, single-photon emission computerized tomography, and positron-emission tomography or structural MR images), fMR imaging is highly user-dependent and requires active participation from both the patient and the investigators throughout the pro- 
cess. Here we will focus on fMR imaging in the clinical setting and will not consider other advanced neuroimaging techniques such as spectroscopy, perfusion, diffusion, or diffusion tensor imaging that might also be relevant to neurosurgical planning.

\section{DATA COLLECTION}

\section{Experimental Population}

Experimental studies investigating fMR imaging have traditionally used undergraduate, graduate, and medical students, or paid healthy volunteers. These populations have generally been eager to participate in experimental studies involving fMR imaging; they understand the instructions and goals of the study, and they are compliant with respect to movement issues in the magnet. However, a patient population is often more heterogeneous with respect to a host of variables such as education, socioeconomic factors, compliance issues, or current disease state. Indeed, patient compliance may be problematic for reluctant participants who may have a number of fears and concerns with respect to their disease state, their prognosis, and what an fMR imaging study actually entails. Additionally, some patients may have considerable difficulty understanding or even performing the task(s) that they are instructed to perform while in the magnet. Head and body movement, falling asleep in the machine, claustrophobic reactions, and cognitive deficits secondary to the disease state as well as difficulties hearing instructions or prompts over the machine noise may have an impact on the reliability of the data derived from an fMR image. ${ }^{10}$

\section{Imaging Techniques}

Functional imaging requires the rapid acquisition of thousands of images. Multislice echoplanar imaging is one of the more commonly used techniques, but other methods may also be used (for example, spiral imaging). Each of these methods has its respective advantages and disadvantages. ${ }^{2,8,19}$ For example, echoplanar imaging is very sensitive to head motion, and individual centers use different methods to stabilize the head. We use a combination of foam pads, a Velcro strap across the forehead, and specific instructions to the patient to remain as motionless as possible during image acquisition. In addition, timing and proper performance of tasks during acquisition of functional images is a critical issue. At our site, all functional imaging sessions are conducted by the investigators, whereas at some sites the use of MR technicians is mandated for all imaging sessions. The addition of functional images into routine clinical procedures would require specific training of the MR technicians or the presence of an investigator during image acquisition.

\section{Paradigm Design}

The critical nature of the timing in FMR imaging studies is due in part to the lag in the hemodynamic response (typically several seconds behind the actual electrical response), ${ }^{2,11,19}$ and the timing has important implications for postprocessing and statistical analysis of the data (see later discussion). When using blood oxygen level-dependent fMR imaging, comparisons of brain regions are made with- out the use of exogenous contrast agents. The comparisons may be of "performance" minus "rest" or "Task 1" minus "Task 2." The task or tasks performed and the timing of the epochs represents the paradigm design. It is critical both that the task(s) reflect the question the clinician seeks to address and that the patient can perform the task(s). These paradigms typically involve either block or event-related designs, and they have been reviewed elsewhere. ${ }^{8,10}$

Our interest is in the integration of fMR imaging within standard neuroimaging procedures that are used for routine clinical examinations. To achieve this goal, our recent work has focused on testing what constitutes a minimal functional data set for assessment of blood flow to a specific brain region for presurgical planning. The goal of these experiments was to facilitate patient compliance in the MR unit by requiring shorter imaging times as well as to decrease the size of the raw data sets that must be stored and processed. We are developing optimized paradigms to assess the functional acquisition during routine clinical imaging, decrease the effect of head motion in the device, and minimize the amount of data collected for motor, language, and auditory systems.

When we initially began using fMR imaging, we routinely performed 6-minute block-design sessions in patients to assess function. Nevertheless, we later found that a shift from 6-minute to 4-minute data sets produced similar results (unpublished observations). In an effort to assess the effects of shorter imaging times on data sets, we have tested healthy volunteers and examined the effects of truncating these 4-minute imaging sessions to the equivalent of a 3 -minute and then to a 2-minute session. ${ }^{5}$ Our findings indicate that three minutes of data acquisition consisting of 30 seconds of rest alternating with 30 seconds of movement may provide sufficient resolution for presurgical mapping; 2 minutes of data acquisition performed using a similar paradigm can provide a general suggestion of brain activation areas, but the resolution is generally poorer compared with 3- and 4-minute sessions. Additionally, we have investigated the length of the epochs in the block-designed tasks (for example, 12 or 30 seconds of movement). ${ }^{9}$ These data indicate that 12 seconds of activity in the dominant hand is able to produce discrete and intense unilateral activation. This activation may represent less blood flow because of the shorter task or increased dexterity in the dominant hand; 30second tasks activated more neurons, resulting in a larger blood oxygen-dependent level response. Identical tasks in the nondominant hand produced more bilateral activation, which could be interpreted as a function of less-practiced motor behavior.

\section{DATA ANALYSIS, INTERPRETATION, AND PRESENTATION}

\section{Data Analysis}

Although data analysis represents the second phase in acquiring and producing an fMR imaging activation map, it is the most labor-intensive and time-consuming step. Unlike standard structural MR imaging, an activation map or fMR image is not immediately available on the technologist's workstation. Instead, the many thousands of "raw functional" images $(\geq 0.5 \mathrm{~GB})$ are transferred offline to a special- 


\section{Bioinformatics and functional magnetic resonance imaging}

ized workstation(s) or server for an analyst to process the data that have been acquired. There are several software packages currently available for analysis of functional neuroimages. These processing tools include commercially available products, freeware, and homegrown, site-specific software that are often referred to with abbreviations. The packages include SPM (Statistical Parametric Mapping), FSL (FMRIB Software Library), AFNI (Analysis of Functional Neuroimages), LIPSIA (Leipzig Image Processing and Statistical Inference Algorithms), BrainVoyager, MedX, Analyze, and VoxBo ${ }^{15}$ (FÅ Neilsen, et al., unpublished manuscript). The SPM, VoxBo, and MedX programs run within MatLab, so a MatLab license is required to execute these programs.

Additional software tools may be used for image processing and subsequent display, depending on the paradigm. Furthermore, in a patient population with "non-normal" brains there are a number of issues with regard to analyzing data in any of these programs. Most programs are geared toward processing data primarily from brains without lesions, pumps, shunts, or other devices commonly found in neurosurgically treated patients. These devices can cause artifacts in the preprocessed data that cannot be corrected by any technique available at the moment. Also, most nonsurgical investigations tend to group data, which requires the normalization of data and its merger onto a "generic" brain. For presurgical planning it is essential that the patient's activation be mapped onto his/her own "nonnormal" brain in an accurate and robust manner.

\section{Data Interpretation}

In addition to the difficulties in data analysis just discussed, interpretation of fMR imaging results in these patient populations presents additional challenges. The mere physical presence of a brain tumor or fluid may shift brain activation patterns. ${ }^{13}$ Increasingly, reports of alterations or translocations of activation ${ }^{16}$ are appearing in the literature. Nevertheless, alterations in or apparent translocation away from lesions and surrounding areas due to neurovascular uncoupling ${ }^{22}$ or changes in hemodynamic response due to cerebrovascular disease ${ }^{3}$ add another consideration in the interpretation of fMR imaging activation maps. Roux, et al., ${ }^{20}$ cautioned that even if the results of preoperative fMR images and the cortical intraoperative stimulations are negative, functional tissue could remain in the peritumoral cortex, and this should be taken into account during the planning phase of a tumor resection. Addressing these issues is critical for the development of fMR imaging for clinical applications. ${ }^{8}$

Another growing body of work investigates fMR imaging and the Wada examination for language localization. The literature is rich and too extensive for an in-depth consideration here. It should be said, however, that caution must be exercised with the use of the Wada examination and fMR imaging. ${ }^{6}$ In our experience, a Wada examination was performed twice and the results clearly indicated bilateral activation; however, the fMR images clearly revealed unilateral activation. On closer examination of the raw preprocessed functional images, an artifact caused by the placement of a shunt blacked out one hemisphere for the entire acquisition period (unpublished data). The question of how to deal with some of these issues is an active area of research. ${ }^{10,12}$
Finally, any interpretation of an fMR image should be made with the knowledge of the task paradigm and the quality of the study performed. It is not sufficient to say this is a 'motor' map. One should ask whether the map was produced with the motion of a single digit, the whole hand, or the arm. Did the language paradigm involve a verb generation task or more complex language tasks? Did the paradigm test receptive or expressive language areas or did it assess both? A record of the amount of patient motion, compliance with instructions, and other possible sources of artifacts needs to be made in case these factors affect the accuracy of the activation maps.

\section{Data Presentation}

In the typical research environment, activation maps are viewed on a workstation display monitor or color images are printed. This method is not optimal or acceptable, however, for routine clinical use in today's environment. The postprocessed fMR images need to be imported into the clinical database or exported to a surgical navigational system, depending on the requirements of the individual case. Unfortunately, neither of these choices is seamless because the postprocessed fMR image is not in the Digital Imaging and Communications in Medicine format, representing additional challenges for the investigator. , $^{1,14,21}$

\section{Management of Data}

Data management is essential in the ongoing acquisition and processing of fMR imaging data. In this section, we consider two possible approaches to this phase of fMR imaging. We currently use a networked server-based laboratory, the MAIAL (Fig. 1) for data analysis. As our group grew over time from three users (two with Ph.D.s and one M.D.) in one location to more than 30 users (faculty, students at all academic levels, and research assistants) across the institution, so did our data processing needs. To accommodate multiple users in multiple locations working in diverse aspects of fMR imaging, we opted for a single-server-based network. This server hosts the high-end and expensive software packages in addition to running a RAID 5 storage system (currently $0.5 \mathrm{~TB}$ ). Site-specific and widely available software is also accessible. This server is housed in the same facility as other hospital computer systems such as the Picture Archiving and Communication Systems. Terminals in the lab and in various buildings across campus provide easy access for the users. Our server supports different vendors' desktops and laptops, but firewall issues must be addressed for offsite access according to the Health Insurance Portability and Accountability Act of 1996.

There are numerous advantages to a server-based approach for data processing, analysis, and management. Foremost among them is the ease of maintenance and accessibility for users. With a central server, the majority of the hardware and software maintenance is done in one location, rather than at multiple workstations. Cost savings is another advantage of this model because it is less expensive to purchase a single license for multiple users than multiple individual licenses that often include additional associated maintenance costs. New users also do not need to purchase and maintain their own systems. Data may be archived on 


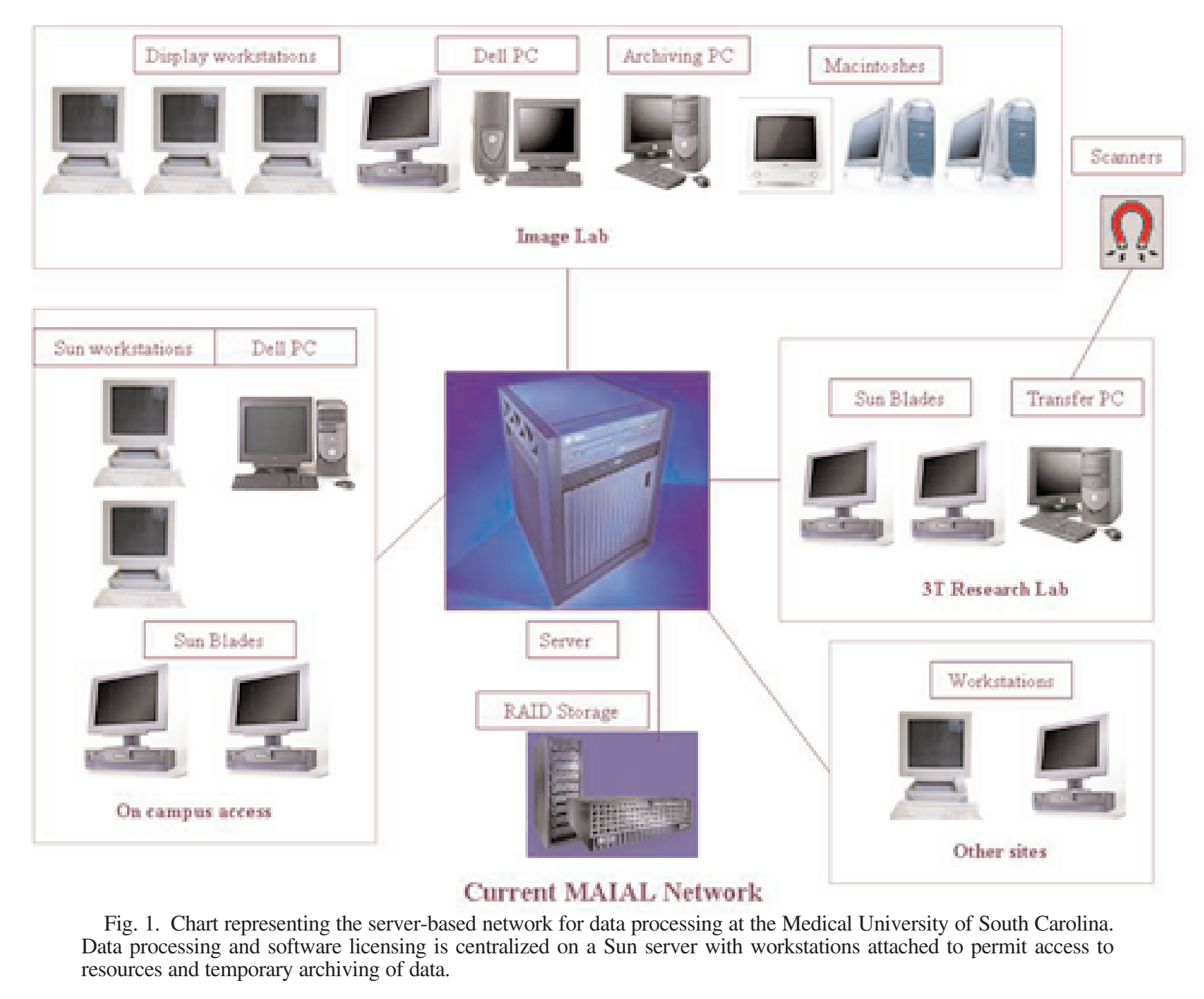

the RAID system or by using hospital resources for longterm storage. Despite those advantages, however, this approach is dependent on a network maintained by the institution; when the network is unavailable, data processing is not possible.

Processing speed was a significant advantage when we first set up the server-based system. With the rapid development of faster desktop workstations, however, this became a less significant advantage. Workstation or laptopbased data analysis is now possible if one has the time and technical skills or if there are people available who are sufficiently adept technically to manage data on a small scale. A high-end desktop system with a significant amount of random access memory can be used for data analysis and management, provided that the machine has been configured with sufficient hard drive space.

The documentation for the software tools described earlier for data analysis should be consulted before use, but most software packages will run in a Linux operating system and some of the software will also run on Macintosh OS X. A variety of versions of Linux are available and some may be freely downloaded. For Windows users, it is possible to configure a machine to dual boot the Windows (2000 or XP) operating system and the Linux operating system. However, additional drive partitioning software is necessary to accomplish this. For other users who require extensive mobility, a high-end laptop may also be used for data processing, provided the central processing unit is sufficiently fast and is configured with enough random access memory to accommodate fMR imaging data sets.

Data analysis generates a significant number of subdirectories and files, so it is important to consider directory structure before data processing begins. Additionally, once processing is complete, data will need to be stored on another large internal drive or an external portable drive. A number of manufacturers produce external hard drives, or one can purchase a drive enclosure and hard drive separately. Alternatively, data may be stored on optical media (compact discs or digital versatile discs).

\section{CONCLUSIONS}

The development of fMR imaging for clinical populations and neurosurgical planning is likely to become more 


\section{Bioinformatics and functional magnetic resonance imaging}

prominent in the future. Numerous challenges lie ahead for the increased use of fMR imaging within a clinical population. Acquisition, postprocessing, interpretation, and management of the data acquired must be coordinated to facilitate access to images for surgical planning and postoperative care. This process can be facilitated by considering the practical issues associated with the bioinformatics of fMR imaging.

\section{Acknowledgments}

We thank Debra Axness, J. Matthew Price, and Alden Finlayson for hardware and software support.

\section{References}

1. Arthurs OJ, Boniface S: How well do we understand the neural origins of the fMRI BOLD signal? Trends Neurosci 25:27-31, 2002

2. Cacioppo JT, Berntson GG, Lorig TS, et al: Just because you're imaging the brain doesn't mean you can stop using your head: a primer and set of first principles. J Pers Soc Psychol 85: 650-661, 2003

3. Carusone LM, Srinivasan, Gitelman DR, et al: Hemodynamic response changes in cerebrovascular disease: Implications for functional MR imagimg. AJNR Am J Neuroradiol 23:1222-1228, 2002

4. Fernandez G, de Greiff A, von Oertzen J, et al: Language mapping in less than 15 minutes: real-time function MRI during routine clinical investigation. Neuroimage 14:585-594, 2001

5. Floyd R, Vincent D, Hurd M, et al: Timing of limb motor activation of fMRI studies. Neuroimage 26 (Suppl 1):S43, 2005 (Abstract)

6. Hammeke TA, Bellgowan P, Binder J: fMRI: methodologycognitive function mapping, in Henry TR, Duncan JS, Berkovic SF (eds): Functional Imaging in the Epilepsies, Advances in Neurology. Philadelphia: Lippincott Williams \& Wilkins, Vol 83, 2000, pp 221-233

7. Heeger DJ, Ress D: What does fMRI tell us about neuronal activity. Nat Rev Neurosci 3:142-151, 2002

8. Hennig J, Speck O, Koch MA, et al: Functional magnetic resonance imaging: a review of methodological aspects and clinical applications. J Magn Reson Imaging 18:1-15, 2003

9. Hurd MW, Vincent D, Powell MA, et al: Hand and foot representation in normal human brain using $3 \mathrm{~T}$ functional magnetic resonance imaging. Neuroimage 26 (Suppl 1):S33, 2005 (Abstract)

10. Kim PE, Singh M: Functional magnetic resonance imaging for brain mapping in neurosurgery. Neurosurg Focus 15(1):E1, 2003

11. Kwong KK: Functional magnetic resonance imaging with echo planar imaging. Magn Reson Q 11:1-20, 1995

12. Lee CC, Ward HA, Sharbrough FW, et al: Assessment of functional MR imaging in neurosurgical planning. AJNR Am J Neuroradiol 20:1511-1519, 1999
13. Majos A, Tybor K, Stefanczyk L, et al: Cortical mapping by functional magnetic resonance imaging in patients with brain tumors. Eur Radiol 15:1148-1158, 2005

14. Maldjian JA, Listerud J, Khalsa S: Integrating postprocessed functional MR images with picture archiving and communication systems. AJNR Am J Neuroradiol 23:1393-1397, 2002

15. Nowinski WL, Thirunavuukarasuu A: A locus-driven mechanism for rapid and automated atlas-assisted analysis of functional images by using the Brain Atlas for Functional Imaging. Neurosurg Focus 15(1):E3, 2003

16. Petrovich NM, Holodny AI, Brennan CW, et al: Isolated translocation of Wernicke's area to the right hemisphere in a $62-$ year-man with a temporo-parietal glioma. AJNR Am J Neuroradiol 25:130-133, 2004

17. Pouratian N, Bookheimer SY, Rex DE, et al: Utility of preoperative functional magnetic resonance imaging for identifying language cortices in patients with vascular malformations. J Neurosurg 97:21-32, 2002

18. Pouratian N, Sheth S, Bookehimer SY, et al: Applications and limitations of perfusion-dependent functional brain mapping for neurosurgical guidance. Neurosurgical Focus 15(1):E2, 2003

19. Price RR, Allison J, Massoth RJ, et al: Practical aspects of functional MRI (NMR Task Group \#8). Med Phys 29:1892-1921, 2002

20. Roux FE, Boulanouar K, Ibarrola D, et al: Functional MRI and intraoperative brain mapping to evaluate brain plasticity in patients with brain tumors and hemiparesis. J Neurol Neurosurg Psychiatry 69:453-463, 2000

21. Rutten GJ, Ramsey N, Noordmans HJ, et al: Toward functional neuronavigation: implementation of functional magnetic resonance imaging data in a surgical guidance system for intraoperative identification of motor and language cortices. Neurosurg Focus 15(1):E6, 2003

22. Ulmer JL, Krouwer HG, Wade MM, Mueller WM, et al: Pseudo-reorganization of language cortical function in fMR imaging: a consequence of tumor-induced neurovascular uncoupling. AJNR Am J Neuroradiol 24:213-217, 2003

23. Witwer BP, Moftakhar R, Hasan KM, et al: Diffusion-tensor imaging of white matter tracts in patients with cerebral neoplasm. J Neurosurg 97:568-575, 2002

Manuscript received September 1, 2005.

Accepted in final form September 22, 2005.

This work was supported in part by grants from Intel and Sun Microsystems to Dr. Vincent. Additional support came from a University Research Resource Facilities grant to the MAIAL. The research included in this paper was funded in part by the MAIAL and the Murray Center for Research in Parkinson's Disease and Related Disorders at the Medical University of South Carolina.

Address reprint requests to: Diana J. Vincent, Ph.D., Department of Radiology, Medical University of South Carolina, Box 250322, 169 Ashley Avenue, Charleston, South Carolina 29425. email: vincentd @musc.edu. 